\title{
Genetic correlates of protection against HIV infection: the ally within
}

\author{
- L. Piacentini ${ }^{1, *}$, M. Biasin ${ }^{1, *}$, C. Fenizia ${ }^{1} \&$ M. Clerici ${ }^{2,3}$ \\ From the Departments of ${ }^{1}$ Preclinical Sciences, University of Milan; ${ }^{2}$ Biomedical Sciences and Technologies, University of \\ Milan; and ${ }^{3}$ Laboratory of Molecular Medicine and Biotechnology, Don C. Gnocchi ONLUS Foundation IRCCS; Milan, Italy
}

\begin{abstract}
Piacentini L, Biasin M, Fenizia C, Clerici M (University of Milan; and Don C. Gnocchi ONLUS Foundation IRCCS; Milan, Italy). Genetic correlates of protection against HIV infection: the ally within (Review). J Intern Med 2008; 265: 110-124.

Repeated exposure to HIV does not necessarily result in infection and HIV infection does not inevitably lead to the development of the AIDS. Multiple immunological and genetic features can confer resistance to HIV acquisition and progression at different steps in
\end{abstract}

viral infection; a full understanding of these mechanisms could result in the development of novel therapeutic and vaccine approaches for HIV infection. In this review, we focus on the genetic mechanisms associated with resistance to HIV infection and to the progression to AIDS.

Keywords: elite controllers, exposed uninfected individuals, exposed-seronegative individuals, genetic correlates, HIV, long-term non progressors.

\section{Introduction}

Twenty-five years after the discovery of human immunodeficiency virus (HIV), neither a therapy nor a vaccine have been developed to eradicate HIV infection. The most recent AIDS Epidemic Update of the World Health Organization (http://www.unaids.org/en/Know ledgeCentre/HIVData/EpiUpdate/EpiUpdArchive/2007/ default.asp) estimates that more than 33 million people live with HIV infection that 2.5 million contracted the infection in 2007 and that 2.1 million people died as a consequence of AIDS in 2007 alone. This occurs despite the fact that our knowledge of the pathogenic mechanisms that cause AIDS keeps getting more and more detailed. One concept that has clearly emerged as a consequence of such knowledge is that the course of HIV infection is affected by inter-individual variability. This has raised the hope that the study of particular cohorts of individuals with nonclassical responses to HIV disease might represent an optimal chance to identify new therapeutic or vaccine targets.

${ }^{*}$ L. P. and M. B. contributed equally to this article.
As a consequence, intense efforts are currently underway to immunologically and genetically characterize individuals that show resistance to HIV disease; these individuals can be classified in two main groups: highly exposed, but seronegative individuals (ESN) and long-term non progressors (LTNP).

Exposed seronegative individuals do not seroconvert despite multiple and documented exposure to HIV. ESN belong to cohort with different exposure profiles: sex workers, children born from HIV-seropositive mothers, intravenous drug users, health care workers accidentally exposed to the virus, homosexuals or heterosexual subjects that have unprotected sex with their seropositive partners. Since the early ' 90 s, numerous studies have examined such cohorts of ESN in the attempt of defining correlates of protection to HIV infection, but none of the identified immunological and/or genetic mechanism has so far been able to explain this phenomenon fully. Attempts to compare results obtained in different cohorts of ESN have had limited success for a number of reasons and in particular, because a clear definition of who should be 
classified as ESN is lacking. We have adopted a strict set of criteria to define sexually exposed ESN individuals. Thus, an ESN is someone who is not HIV-infected despite a history of multiple unprotected sexual episodes (with an HIV-seropositive partner) for $\geq 4$ years at the time of enrolment, with at least eight episodes of at-risk intercourse within the 4 months before study entry and an average of 30 reported unprotected sexual contacts per year $[1,2]$. A common and widely accepted set of criteria is nevertheless missing and is sorely needed.

Long-term non progressors are a small minority $(<2 \%)$ of HIV-seropositive patients characterized by a long course infection that does not result in signs or symptoms of AIDS, despite the absence of antiretroviral therapy. CD4 $\mathrm{T}$ cell counts are stable and HIV plasma viraemia is low in LTNP. Similarly to what happens for ESN, results obtained in different cohorts of LTNP are difficult to compare, mostly because of the heterogeneity of inclusion and follow-up criteria and because of the diverse technical approaches used by different investigators and centres. Patients with nonprogressive HIV disease were recently sub-classified into two different groups: 'classical' LTNP and 'elite controllers'. Compared to LTNP, who show variable but low viral load, the elite controllers are characterized by a persistently undetectable plasma viraemia $\left(<50\right.$ copies $\left.\mathrm{mL}^{-1}\right)$. Lately, the International HIV Controller Consortium has somewhat relaxed the requirements that define this population of HIVinfected individuals. Thus, by the new definition, elite controllers are HIV-infected patients with HIV RNA levels below 50 copies $\mathrm{mL}^{-1}$ despite the absence of antiretroviral therapy (for at least 1 year at the time of the study). Spikes of viraemia up to 1000 copies $\mathrm{mL}^{-1}$ are accepted in elite controllers, as long as these episodes are not consecutive and represent the minority of all available determinations [3]. For a definition of the different groups of individuals with classic or nonclassic response to HIV infection, see Table 1.

It is actually impossible to predict whether the resistance to disease progression shown by LTNP and elite controllers will last indefinitely or whether progression to AIDS will eventually be detected in these patients. Results from Birch et al. and Goudsmit et al. $[4,5]$ nevertheless seem to indicate that most of the individuals initially considered to be LTNP do eventually develop AIDS. These considerations notwithstanding, the researchers' expectancy is that the efforts spent on these subjects will greatly contribute to clarify the host-genetic traits that characterize nonprogressive HIV disease.

We have recently reviewed the immunological peculiarities of ESN [6]. In this article, we will focus on the peculiar genetic background that seems to be at least partially shared between ESN and LTNP.

\section{Genetic correlates of protection against HIV infection}

It has become clear that genetic variations amongst humans can influence susceptibility to HIV infection and progression to AIDS. Individuals homozygous for the $\Delta 32$ allelic variant of the CCR5 protein, the co-receptor for the macrophago-tropic R5-HIV viruses, display a strong, even if not absolute, protection to sexually transmitted HIV infection [7, 8]. This discovery encouraged several researchers to look for other mutant genes involved in the immune response against HIV. Many of these genes were identified by genetic association studies, but some association analysis - based merely on statistical significance - led to artifactual results. For this reason, criteria for validation have been proposed by several authors (as reviewed by O'Brien) [9]. Such criteria include: (i) the analysis of consistent cohorts of individuals; (ii) statistical correction for multiple tests; (iii) the independent replication of significant associations in different populations, ethnic groups or cohorts; (iv) the establishment of a relationship between the newly identified mutation and the modulation of the gene product resulting in a plausible functional explanation toward AIDS development.

An abundance of data describes a plethora of factors possibly influencing HIV infection and progression to AIDS. For the sake of synthesis, we have chosen to focus our attention on the most significant genetic correlates of protection described to date. These 
Table 1 Immuno-virological characterization of study populations

\begin{tabular}{lllll}
\hline & & & & HIV-1 chronic \\
Parameters & ESN & LTNP & EC & progressors \\
\hline $\begin{array}{l}\text { CD4+ T cell } \\
\text { count }(\text { cells mm }\end{array}$ & $>500$ & $>500$ & $>500$ & $<500$ \\
Viral Load & & & & \\
$\quad$ copies $\mathrm{mL}^{-1}$ ) & & & & \\
HIV-1 specific IgG & - & + & + & + \\
HIV-1 specific IgA & + & + & + & + \\
HIV-1 specific T cells & + & + & + & + \\
Antiretroviral therapy & - & - & - & +
\end{tabular}

ESN, exposed seronegative individuals; LTNP, long term non progressors; EC, elite controllers.

correlates mainly belong to two families: chemokine receptors/chemokine receptor ligands and human leukocyte antigens (HLA).

\section{CCR5 variants}

CCR5 is a polymorphic chemokine receptor expressed on memory/effector T-cells, macrophages, immature dendritic cells and microglia cells [10]. Several structural CCR5 variants have been described; the prevalence of these variants seems to be different in different ethnic groups. The CCR5 32 variant was first identified by sequencing the CCR5 coding region in randomly selected or in HIV-exposed uninfected individuals. The mutant allele bears a 32 bp deletion in a region corresponding to the second extracellular loop of the receptor. This deletion causes a frame shift that gives rise to a truncated and nonfunctional isoform of the wild-type receptor $[7,8]$. Susceptibility to HIV infection is greatly diminished in CCR $5 \Delta 32$ homozygous individuals and PBMC from these individuals are almost uninfectable in vitro by R5-HIV strains $[7,8,11]$. CCR5 432 heterozygous individuals can be infected by HIV, but are characterized by a delayed progression towards clinical stages of AIDS. The phenotypic effect of the CCR5 432 heterozygosis consists in a lower expression of functional CCR5 molecules on the cell surface compared to wild-type homozygosis. This phenotype results in a reduced ability of HIV to bind its co-receptor and, ultimately, in a diminished viral replication. [12-17].
CCR5432 homozygosis does not confer absolute protection against infection. Thus, a number of reports have shown that HIV-infected CCR5 $\triangle 32$-homozygous individuals can be identified proving the incomplete protective power of this genetic variant towards HIV infection [18-24]. The CCR5 32 allele occurs with a frequency of $4-15 \%$ in Caucasian ethnicity (even higher frequencies can be observed in Northern Europeans) and homozygous individuals are overrepresented in ESN. The CCR5 332 variant is nevertheless completely absent in sub-Saharian African populations, where the majority of HIV infection occurs and where many investigators have described numerous cohorts of ESN (Fig. 1). The role of this mutation in the resistance of ESN to HIV infection at a population level seems therefore to be marginal at best [25]. Noticeably, the analysis of CCR5 432 in elite controllers did not reveal any peculiar pattern when results were compared to those obtained in the seronegative population suggesting that this chemokine receptor polymorphism might not be associated with control of disease progression in the absence of therapy [26]. Finally, other mutations in the CCR5 gene have been described; many of them seem to affect the normal co-receptor structure. These variants are very rare and restricted to specific populations and their role in HIV susceptibility has not been thoroughly established [27].

\section{Variants in the CCR5 promoter region}

The observations that: (i) CCR5 expression is highly variable amongst people, and (ii) the levels of CCR5 expression influence in vitro HIV infection [14] led to investigations of the CCR5 promoter variants in the attempt to unveil the genetic background responsible for CCR5 expression. Many CCR5 polymorphisms have been described and some haplotypes have been associated with a different transcriptional regulation and with a different rate of AIDS progression [2830].

Individuals bearing the CCR $5 P 1$ haplotype apparently progress more rapidly to AIDS, even if studies performed in healthy controls bearing or not-bearing this haplotype show comparable levels of CCR5 expression 
Fig. 1 Frequency (\%) of the CCR5 432 allele in Europe and Northern Africa. A north-south gradient is observed and this variant is absent in sub-Saharian African countries.

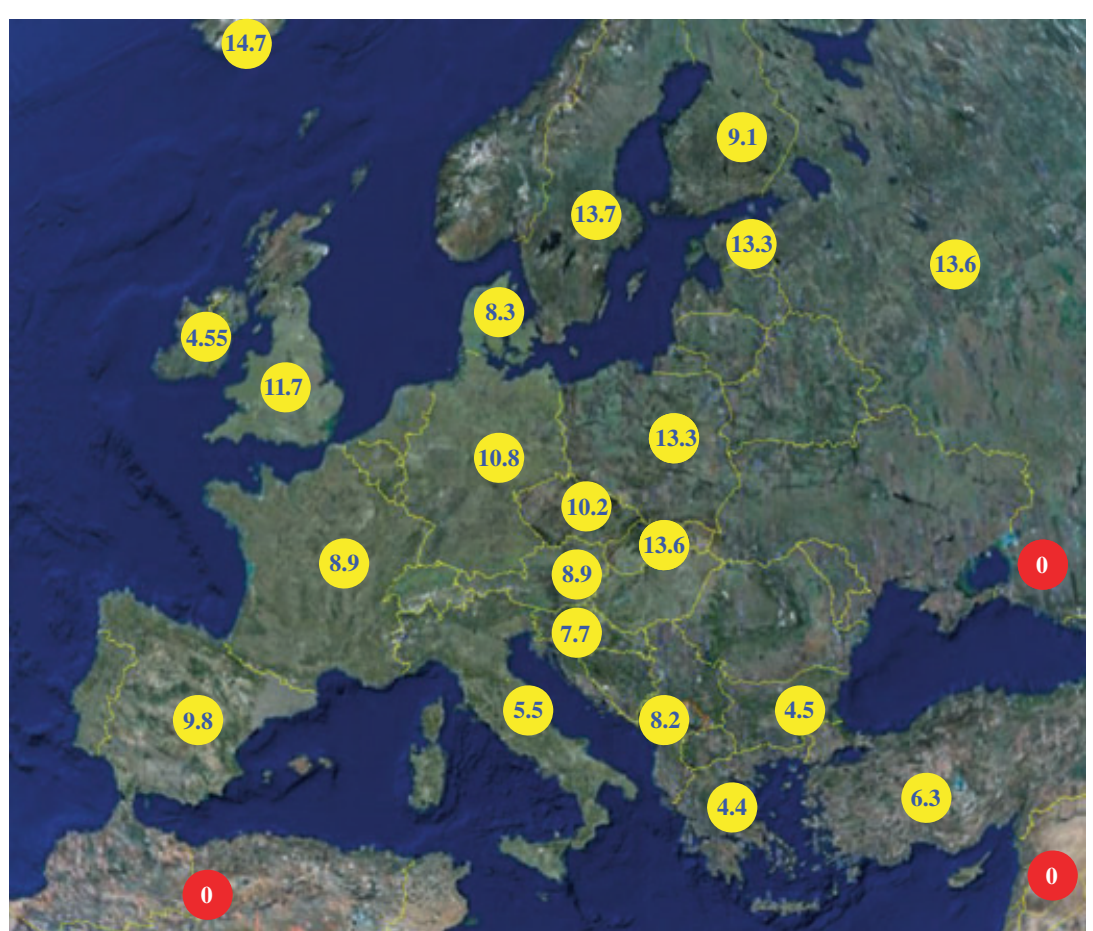

on PBMC of both groups of individuals [31]. Other results indicate that patients possessing the 59029A/A polymorphism, located within the first intron of CCR5, progress more rapidly to AIDS. Noticeably, the $59029 \mathrm{G}$ allele has been associated with lower CCR5 expression on PBMC [32, 33]. Another study showed that the frequency of the 59353C allele was higher in full blown AIDS patients compared to LTNP suggesting a possible role for this mutation in AIDS progression [30]. Finally, the combined effect of the CCR5 432 allele and the CCR5-2459G promoter polymorphism was shown to correlate with poor CCR5 expression and resistance to HIV infection in ESN [34].

\section{Other co-receptor variants}

The CCR2 V64I variant is a mutant allele in the CCR2 gene shown to affect AIDS progression. The mechanism of action and the association of the V64I allele with slower disease progression are controversial and, as CCR2 is a co-receptor used by few HIV strains, it is unlikely that the effect of this mutation alone plays a pivotal role in modulating resistance to
HIV disease [34-36]. Another minor HIV co-receptor is the polymorphic chemokine receptor CX3CR1; this molecule is suggested to influence HIV replication via the recruitment of immunomodulatory cells. Individuals homozygous for the CX3CR1-I249/M280 haplotype progress more rapidly to AIDS compared to subjects bearing different haplotypes [37]. Noticeably, a Spanish study showed that the CX3CR1-I249/T280 haplotype is more frequently detected in LTNP compared to patients with a classical disease progression [38]. Both these correlations need to be confirmed in ampler cohorts of patients.

Recent results [39] focused on a 26-kb locus on chromosome 2q35. This locus harbours the CXCR1 and CXCR2 genes that encode the receptor proteins of the pro-inflammatory chemokine IL-8. Background data for this research project stem from the observation that, whereas there are no established associations between $I L-8$ gene variants and susceptibility to HIV infection, a reduction of CXCR1 and CXCR2 expression is observed in HIV infected patients [40]. Vasilescu et al. thus genotyped rapid and slow progressors of a French cohort and showed that the CXCR1-Ha 
haplotype, carrying the CXCR1_300 and CXCR1_142 $\mathrm{SNPs}$, is less frequent in rapid compared to slow progressors. Transfection of the Jurkat and CEM T cell lines with cDNA encoding the CXCR1-Ha haplotype led to a reduction in CD4 and CXCR4 expression levels. Moreover, when these transfected cells were challenged with the X4-HIV $\mathrm{NL4-3}_{3}$ strain, decreased infection efficiency was detected when CXCR1$H a$-transfected cells were compared to cells transfected with the wild type allele cDNA. These elegant experiments led to the conclusion that the $\mathrm{CXCR} 1-\mathrm{Ha}$ haplotype is associated with a reduced expression of CD4 and CXCR4 resulting in a lower infection efficiency of X4-tropic strain. Noticeably, because both CD4 and CXCR4 are essential factors in cell development and functionality, the consequence of a marked reduced expression of these proteins on cell physiology needs to be thoroughly investigated [39].

\section{Chemokine variants}

A number of studies dissected the possible role of CC-chemokines in the modulation of susceptibility to HIV disease. These chemokines - RANTES/CCL5, MIP- $1 \alpha / C C L 3$, MIP- $1 \beta /$ CCL 4 and MIP $1 \alpha$ P/CCL3L1 - are highly related CC proteins that bind CCR5 and are capable of suppressing HIV replication [7, 41]. The effect of CC chemokines on HIV is secondary to their interaction with CCR5 and is mediated by either a direct chemokine-virus competition for the receptor or by the induction of receptor internalization [14].

High RANTES levels were observed in ESN and in HIV-infected individuals with a slow rate of disease progression [42-45] suggesting a role for RANTES in preventing or limiting HIV disease. A possible explanation for the differences detected in RANTES expression levels comes from the identification of genetic variants in its promoter region. Thus, the haplotype defined by the SNPs $-403 \mathrm{G} / \mathrm{A}$ and $-28 \mathrm{C} / \mathrm{G}$ in the RANTES promoter region was associated with a slower decrease in CD4+ T-cell count in Japanese and Thai cohorts of HIV-infected individuals [46, 47]. In another study, the RANTES $-408 \mathrm{~A}$ variant was associated to a delayed clinical progression of HIV infection. Noticeably, this same variant was also shown to be a risk factor for HIV transmission [48]. These effects were nevertheless not confirmed by other studies and, thus, remain ambiguous. Another SNP, In1.1C, located within an intronic regulatory region of the RANTES gene, is associated with the down-regulation of RANTES production. Accordingly, HIV-infected patients bearing this allele progress more rapidly to AIDS [49]. Overall, these data support the idea that RANTES may have an important role in modulating HIV disease.

CCL3L1, another CC chemokine, is considered the most potent agonist of CCR5 and the most effective inhibitor of R5-HIV strain. The gene for this chemokine, together with those for CCL3, CCL4 and CCL5, arose from the duplication of a common ancestral gene and nonallelic copies are found in variable numbers in the human population. Gene duplication can result in overproduction of the corresponding protein; in turn, the protein overabundance may optimally modulate effective immune responses. Gonzales et al. studied the relationship between CCL3L1 copy numbers and susceptibility to HIV infection in a large cohort of HIV-infected and -uninfected individuals of different ethnicities. Results showed that CCL3L1 secretion levels are directly associated with gene copy number and inversely related to CCR 5 expression on CD4+ T-cells [50]. These relationships might explain the mechanism by which chemokines exert their protective role. Thus, down-regulation of the expression of CCR5 on cell surface would make the binding of this protein by HIV more difficult, effectively hampering HIV infectivity. Gonzales et al. also observed that lower copy numbers of CCL3L1 are present in HIVinfected compared to uninfected individuals. Noticeably, the authors indicated that HIV susceptibility is not strictly related to the absolute gene copy number, but it depends on copy numbers carried by an individual compared to other individuals of the same population. In other words, the median CCL3L1 copy numbers bore by the whole population could be considered a threshold over which protection against HIV infection is favoured [50].

The prominent role-played by CC-chemokines in modulating HIV disease and their close location on 
chromosome 17, stimulated the analysis of genetic variation in chemokine gene clusters. Modi et al. genotyped 21 SNPs in a 47-kb segment on chromosome $17 \mathrm{q} 12$ - where CCL3, CCL4 and CCL18 genes map - in five different cohorts of American HIVinfected or HIV-exposed uninfected individuals. Results showed that an extensive linkage disequilibrium (LD) in a $37-\mathrm{kb}$ segment containing 17 SNPs is present in Americans of both Caucasian and African ethnicities. Two main associations were also reported: (i) three correlated SNPs in CCL3 gene are more frequent amongst ESN compared to HIV infected individuals; (ii) seven related SNPs, spanning a 36-kb region that contains the three chemokine genes, are significantly associated with rapid progression amongst Americans of Caucasian ethnicity [51]. Even though the authors did not identify a functional mutation, this association study reinforces the idea that variations in chemokine gene could play a role in modulating chemokine levels and thus resistance to HIV disease and/or AIDS progression.

A similar analysis was also performed by the same authors on another gene cluster that is located $1.8 \mathrm{Mb}$ upstream the previous one. In this study, extensive LD was also observed and the CCL2-CCL7-CCL11 $H 7$ haplotype was shown to be significantly more frequent in sexually exposed ESN of Caucasian ethnicity. As the proteins encoded by these genes are not ligands for CCR5 or CXCR4 and, thus, do not modulate virus entry, the authors proposed that a purely immune mechanism, i.e. the chemokine-mediated recruiting of monocytes, eosinophils and dendritic cells to the inflammation site, could mediate the protective effect of the $H 7$ haplotype [52].

The important role of CXCR4 as a co-receptor for X4 HIV strains in the more advanced stage of HIV infection led to investigations focusing on SDF-1, the natural ligand of he CXCR4 protein. The SDF-1 3'A variant stems from a point mutation in the $3^{\prime}$ untranslated region of the SDF-1 mRNA. This variant increases transcript stability and, thus, enhances SDF1 production [53]. The SDF-1 $3^{\prime} A$ variant is present in variable frequencies amongst the population-atlarge and, when present in a homozygous condition, is suggested to be associated with delayed onset of AIDS. This protective effect was not observed in heterozygous individuals and is presumably caused by the direct competition between SDF-1 and the X4 HIV strains for binding to CXCR4. Noticeably, subsequent studies did not confirm this result [54] and meta-analysis of different cohorts seem to reinforce the idea that there is not a real effect of the $S D F-1$ $3^{\prime} A$ variant on AIDS progression [55]. For a summary of the different chemokine receptors and chemokine ligands genes variants, see Table 2 .

\section{Human leukocyte antigen (HLA) polymorphisms}

Human leukocyte antigen genes are highly polymorphic loci encoding for cellular surface molecules that are responsible for antigen presentation to $\mathrm{T}$ lymphocytes. The efficiency of this process differs amongst individuals because of the high number of allelic variants of HLA class $I$ ( $A, B$ and $C$ ) and class II genes (DR, DQ and DP). The HLA class $I$ genes encode molecules that bind antigens derived from the processing of intracellular pathogens and present these antigens to cytotoxic $\mathrm{T}$ lymphocytes (CTL). In contrast, HLA class II genes produce molecules that bind peptides of extracellular origin and present them to CD4+ $\mathrm{T}$ helper lymphocytes. Individuals expressing the most polymorphic $H L A$ variants and those variants with the best ability to bind to exogenous peptides, mount a more vigorous immune response against pathogens.

Several reports describe associations of particular HLA alleles with different outcomes of HIV infection, but in many instances, these observations were not confirmed by subsequent studies. These studies are difficult to perform as the results need to be validated in large cohorts and need ethnic-background stratification, precise clinical information and a broad knowledge of the disease genetic; lack of any of these factors will weaken the obtained results [56]. These caveat notwithstanding, many studies report solid, confirmed associations between given HLA molecules and different patterns of HIV disease; we will summarize the most compelling of these studies. 
Table 2 Chemokine receptors and ligands gene variants involved in HIV-1 resistance and/or AIDS progression

\begin{tabular}{lll}
\hline Gene variants & Associated to & Mechanism of action \\
\hline CCR5 32 & Resistance and delayed progression & Abrogated or decreased CCR5 cell \\
& & surface expression [7, 8, 11-17] \\
CCR5P1 & Accelerated progression & Increased CCR5 expression [31] \\
CCR5 59029AA & Accelerated progression & Increased CCR5 expression [32, 33] \\
CCR5 59353C & Accelerated progression & Increased CCR5 expression [30] \\
CCR5 $32 / C C R 5-2459 \mathrm{G}$ & Resistance & Reduced CCR5 expression [34] \\
CCR2 V64I & Delayed progression & Influence CCR5 or CXCR4 expression(?) [27] \\
CX3CR1 I249/M280 haplotype & Accelerated progression & Impaired fractalkine binding [37, 38] \\
CCL5 (RANTES) -403G/A and $-28 C / G$ & Delayed progression & Increased CCL5 expression [46, 47] \\
CCL5 (RANTES) In1.1C & Accelerated progression & Decreased CCL5 expression [49] \\
CCL3L1 (high copy numbers) & Delayed progression & Higher CCL3L1 expression [50] \\
Chromosome 17q12 haplotype & Resistance & Modulation of chemokine levels (?) [51] \\
Chromosome 17 haplotype H7 & Resistance & Stimulation of the immune response [52] \\
CXCR1-Ha haplotype & Delayed progression & Reduced expression of CD4 and CXCR4 [39] \\
SDF-1 3'A & Delayed progression & Enhanced SDF-1 production (?) [53] \\
\hline
\end{tabular}

A vast $H L A$ polymorphism is suggested to be associated with a more efficient immune response against HIV. This effect results from the ability of individuals expressing such polymorphisms to present a wider repertoire of antigens to $\mathrm{T}$ cells hindering the appearance of HIV escape mutants. Carrington et al. tested this hypothesis in a cohort of 498 HIV infected individuals and demonstrated a highly significant association between HLA class I homozygosity and rapid progression to AIDS in American individuals of both Caucasian and African ethnicity [57]. Similar results were also obtained when $H L A-A$ and $H L A-B$ loci were analysed in infected Dutch homosexual men as well as in Rwandan heterosexual women [58]. The take home message from these analyses is that HLA homozigosity is associated with faster progression of HIV disease.

Data obtained in independent studies involving different cohorts of patients have convincingly shown that correlations also exist between the HLAB*57 and $B^{*} 27$ molecules and delayed AIDS progression; conversely, the $B^{*} 35$ allele is associated with a more rapid disease progression [56]. The protective effect of HLAB57 was shown in several LTNP studies in which the authors highlighted the ability of HLAB57- restricted CTL to target multiple HIV peptides. The B5701 and the B5703 alleles (present mostly in Caucasians and Africans respectively) exhibit a broad cross-reactivity against both common and rare variants of dominant gag epitopes and have been associated to delayed progression of HIV disease [59-61]. Elegant analyses showed that the protective effect of the HLAB27 allele is dependent on the high stability of the interaction between the HLA B pocket and the arginine in position 2 of the gag peptide. In fact, mutations in this position lead to both unsteadiness of the B27-gag complex and faster progression to AIDS $[62,63]$. The HLA class I Bw4 homozygosis has also been associated with delayed progression to AIDS and with the maintenance of normal CD4+ -cell count in LTNP [64]. Moreover, it has been shown that the interaction between HLA Bw4 and the corresponding KIR3SD1 receptor on natural killer cells suppresses HIV-infected lymphocytes resulting in a positive effect on disease progression [65].

Interestingly, the $H L A B^{*} 57$ and $B^{*} 27$ above mentioned polymorphisms were shown to be over-represented in elite controllers compared to patients showing a normal disease progression. Nevertheless, $<50 \%$ of these elite controllers show the presence of 
HLAB*57 and an even smaller percentage (20\%) express HLA-B27, the other widely accepted protective HLA molecule [26]; these frequencies are much lower than that the one previously reported in smaller cohorts $[66,67]$. In the same study, $68 \%$ of elite controllers, but only $37 \%$ of patients with normal disease progression, were shown to carry at least one of the HLA alleles (B5801, HLA B27, B1503, B13 and B51) associated with delayed disease progression. Noticeably, these results indicate that almost one-third of elite controllers do not express any of the known protective $H L A$ alleles. Therefore, although these findings clearly suggest an association between given HLA genotypes and the control of viral replication, other factors must be involved in determining the favourable disease outcome that characterizes these patents.

A role for $H L A$ genes in determining resistance toward HIV infection has also described in HIV-sexually exposed seronegative individuals including healthy newborns of HIV-infected mothers. The foremost observation is that the concordance of HLA class I alleles between the HIV infected subject and the uninfected sexual partner results in an increased likelihood of HIV sexual transmission [68, 69]. Analyses performed in pregnant mothers in Nairobi also showed that the sharing of HLA class I alleles between mothers and their newborns increases the risk of perinatal HIV transmission [70]. These data seem to indicate that wider differences between the HLA repertoire of the HIV-infected patient and of the HIV-uninfected individual result in the generation of highly heterogeneous and possibly stronger immune responses that decrease the likelihood of viral transmission.
Clustering of HLA molecules in supertypes characterized by functional and structural similarity also allowed the detection of an association between the HLA A2/6802 supertype molecule and a decreased risk of seroconversion amongst commercial sex workers in Kenya [71]. A similar result was obtained in a mother to infant cohort in Nairobi: in this study, the same HLA supertype was associated with a reduced perinatal HIV infection risk [72].

Finally, other HLA molecules are associated not with protection, but rather with faster progression of HIV disease. Amongst such molecules, HLA-B*35 plays a predominant role. Thus, the $B^{*} 35-C w^{*} 04$ haplotype was significantly associated with disease progression in Caucasians, but not in African-American [57]; a subsequent study showed a clear association of the $B^{*} 35-P x$ subtype allele with accelerated AIDS progression [56]. The explanation proposed for this association is the very reduced capacity of the $B^{*} 35-P x$ allele to bind HIV peptides resulting in its consequent inability to raise an effective virus-specific immune response in infected individuals. Moreover, it has been demonstrated that $B^{*} 35-P x$-heterozygous individuals progress more rapidly to AIDS compared to individuals homozygous for any other allele of the $H L A-B$ gene. The authors suggest that the $B^{*} 35-P x$ allele yields a negative effect on HIV pathogenesis [56] (Table 3).

On the whole, these data indicate that even though some HLA alleles are more represented in ESN, LTNP and elite controllers, other, still unknown host factors or combination of factors contribute to these remarkable phenotypes.

Table 3 HLA gene variants involved in HIV-1 resistance and/or AIDS progression

\begin{tabular}{lll}
\hline Gene variants & Associated to & Mechanism of action \\
\hline HLA class I heterozygosity & Delayed progression & Broader HLA class I antigen presentation [56-58] \\
HLA class I discordance & Decreased frequency of infection & Diminished risk of HIV transmission [68-70] \\
HLAB*57 & Delayed progression & Delayed HIV-1 escape [26, 56, 59-61] \\
HLAB*27 & Delayed progression & Delayed onset of escape mutants [26, 56] \\
HLAB*35 & Accelerated progression & Reduced HIV peptides binding [56] \\
HLA supertype A2/6802 & Resistance & Presentation of conserved HIV peptides [71, 72] \\
HLA class I Bw4-KIR3SD1 & Delayed progression & Suppression of HIV infected lymphocytes (?) [65]
\end{tabular}

*allele-group gene products. 


\section{Endogenous antiretrovirals}

The ability to modulate HIV replication has recently been associated with mechanisms other than chemokines and HLA proteins as well. Thus, host endocellular factors modulating postentry events of HIV replication have moved into the spotlight in recent years. The most interesting of these factors are APOBEC3G and TRIM5 $\alpha$, proteins belonging to the cytidine deaminases and to the tripartite motif family respectively.

The host endocellular factor APOBEC3G is endowed with a broad antiviral activity and is believed to contribute to resistance to HIV infection. APOBEC3G was shown to reduce the fitness of virion infectivity factor (Vif) protein-deleted strains of HIV, thus preventing viral cDNA integration in the cell genome. This effect was shown to be mediated by the cytidine deamination of viral reverse transcripts and/or by mechanisms independent from this enzymatic activity. The antiviral activity of APOBEC3G in wild-type viruses is counteracted by Vif; the resulting APOBEC3G-Vif bimolecular complex is then degraded via the ubiquitine-proteasome pathway [73]. These observations have triggered a series of studies in the attempt to identify possible genetic variants of the APOBEC3G gene that might encode for a mutant protein able to overcome Vif mediated degradation, thus conferring resistance to HIV disease. A variety of SNPs have been identified in the APOBEC3G gene, whereas none of these has been associated with resistance; some SNPs are clearly over-expressed in patients with faster disease progression. Thus, the $186 \mathrm{R}$ allele, which is significantly more present in African Americans (37\% compared to 3\% in Americans of Caucasian ethnicity), is strongly associated with a faster decline of $\mathrm{CD} 4+\mathrm{T}$ cells and accelerated progression to AIDS [74]. Analogously, the allele $C 40693 T$ is associated with increased risk of infection in ESN and faster disease progression in HIV infected individuals [75].

A number of in vitro results seem to indicate that different expression levels of wild type APOBEC $3 \mathrm{G}$, rather than variations in the protein structure, could play an important role in altering the course of HIV infection. If this is the case, changes in the quantity, rather than in the quality, of APOBEC $3 \mathrm{G}$ would be correlated with different outcomes of HIV infection in vivo. Accordingly, it was demonstrated that even slight changes in APOBEC3G expression result in decreased viral replication [76]. In a work performed in our laboratory, Biasin et al. evaluated APOBEC3G expression in $\mathrm{PBMC}$ and in $\mathrm{CD}^{+}, \mathrm{CD} 8+$ and CD14+ cell subsets from ESN, HIV-infected subjects and healthy controls. Results showed that higher basal and IFN $\alpha$-stimulated APOBEC $3 \mathrm{G}$ mRNA and protein levels are seen in ESN compared to the other two groups. Furthermore, in vitro infection assay with the

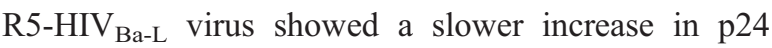
concentration and a faster increase in APOBEC $3 \mathrm{G}$ levels in PBMC from ESN compared to healthy controls. The different kinetics of p24 and APOBEC3G expression might be explained by the fact that ESN would respond to IFN $\alpha$ - one of the first cytokines produced early in viral infections - with a faster and more robust production of APOBEC3G. This, in turn, would limit HIV replication. Interestingly, CD14+ monocytes of ESN were observed to be more responsive to IFN $\alpha$ stimulation. These results suggest the possibility that monocytes-derived cells, such as Langerhans cells and macrophages, might express higher amount of APOBEC3G as well, possibly constituting an important barrier to HIV infection in peripheral tissue [77]. As no effective mutations have been detected in the $A P O B E C 3 G$ promoter, these data seem to confirm that APOBEC $3 \mathrm{G}$ exerts its antiviral role by varying its expression (possibly following immune modulation by cytokines, such as IFN $\alpha$ ). Further support to this concept stems from data by Jin et al. [78] showing correlations between APOBEC3G levels and CD4+ T cell counts and viraemia in a cohort of LTNP indicating a role of this protein in the control of HIV disease progression (Fig. 2).

A second antiviral factor, TRIM5 $\alpha$, was recently shown to restrict the replication of a broad range of retroviruses through its interaction with the capsid of such viruses [79]. TRIM5 $\alpha$ restricts retroviruses in a species-specific manner as, for example, HIV replication is blocked by simian TRIM5 $\alpha$ alone. 
Fig. 2 Exon distribution and genomic locations of the SNPs associated with resistance to/progression of HIV infection in the $A P O B E C 3 G$ and TRIM5 $\alpha$ genes. The SNPs are numbered according to the first base of reference sequence AL022318 for intronic SNPs in $A P O B E C 3 G$, and of the amino acid sequence NP_068594 (APOBEC3G) and NP_149023 (TRIM5 $\alpha$ ).

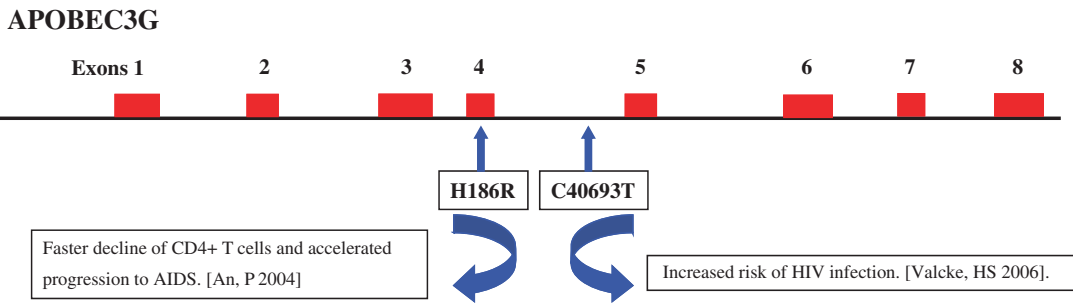

TRIM5 $\alpha$

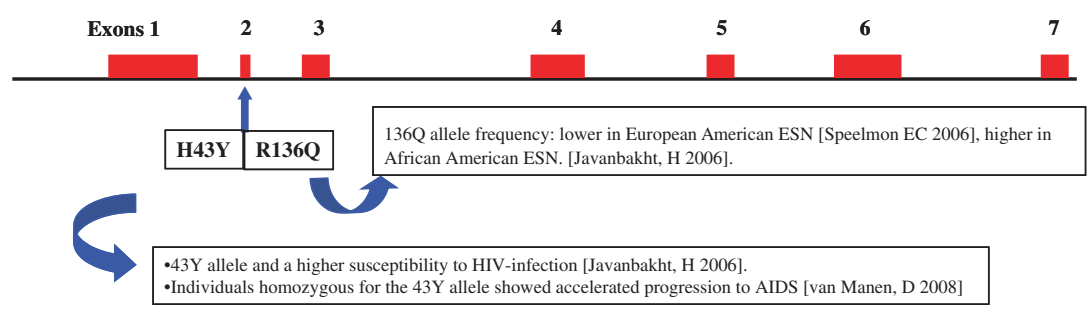

Physiological levels of human TRIM5 $\alpha$ slightly impairs HIV replication and some authors have suggested that polymorphisms in the TRIM5 $\alpha$ gene might be associated with a higher anti HIV activity. To this end, the 136R (R136Q polymorphism) and the $43 \mathrm{H}$ (H43 polymorphism) alleles were found to be associated with in vitro resistance to HIV infection. Noticeably, the 136Q allele was found to be significantly more frequent in HIV-infected individuals compared to ESN of Caucasian ethnicity [80]; the same allele was nevertheless found to be more frequent in African American ESN compared to HIV-infected patients [81]. A different genetic background and different routes of exposure might explain these contradictory results. Another allele, 43Y, was found to be more frequent in the ESN compared to HIV-infected individuals. This result contrasts with in vitro evidences showing an association between this allele and a higher susceptibility to HIV infection [81] (Fig. 2).

Both of these alleles were analysed very recently in an independent cohort of 364 Caucasian homosexual men enrolled in the Amsterdam Cohort. Data stemming from these analyses revealed a possible association between homozygosity for the $43 \mathrm{Y}$ allele and accelerated progression to AIDS (results were compared to those obtained in individuals heterozygous for the $43 \mathrm{Y}$ allele or homozygous for the $43 \mathrm{H}$ allele).
A protective effect of the 136Q genotype after the isolation of X4-HIV strains from the blood of patients was also observed, but no correlation with disease progression was detected. Other results indicated that the co-presence of the $136 \mathrm{Q}$ and of the $-2 \mathrm{GG}$ alleles localized in the $5^{\prime}$ UTR of TRIM5 $\alpha$ is associated with accelerated disease progression [82]. Finally, results obtained in a study analysing the most common human TRIM5 $\alpha$ variants: H43Y, V112F, R136Q, G249D and H419Y failed to show any significant associations between any of such variants and progression of HIV disease [83]. (Table 4).

\section{Other polymorphisms}

The IL-4 gene cluster within chromosome 5 has recently become the focus of interesting genetic analyses. This region harbours several immune genes involved in Th1 and Th2 cellular differentiation, such as $I L-4, I L-3, I L-5, I L-13$ as well as the interferon-regulatory factor $1(I R F-1)$ genes. Ball et al. investigated the possibility that polymorphisms in the IL-4 gene cluster could be related to HIV resistance in ESN Kenyan sex workers. Results of analyses spanning this region showed differences in the allelic distribution of the D5S1984 and IRF-1 microsatellite markers in ESN compared to HIV-infected individuals. Noticeably, the 179 allele at $I R F-1$ microsatellite was 
Table 4 Other gene variants involved in HIV-1 resistance and/or AIDS progression

\begin{tabular}{lll}
\hline Gene variants & Associated to & Mechanism of action \\
\hline APOBEC3G 186R & Accelerated progression & Defect in antiviral activity (?) [74] \\
APOBEC3G C40693T & Increased risk of infection & Absence of functional APOBEC3G (?) [75] \\
TRIM5 $136 Q$ & Resistance to X4 HIV-1 & Affect protein oligomerization and antiviral activity (?) [80, 81] \\
& strains/susceptibility & \\
TRIM5 $43 Y$ homozygosity & Resistance/susceptibility & Affect protein structure and antiviral activity (?) [81, 82] \\
IRF-1 619A/6516G & Resistance & Lower IRF-1 expression and HIV-1 replication [98] \\
Chromosome 22q13 & Resistance & Strong immune response against HIV-1 [90] \\
microsatellite markers & & \\
& & \\
\hline
\end{tabular}

associated with the resistant phenotype; the presence of at least one copy of 179 allele was also shown to be associated with a decreased risk of seroconversion. Further results focusing on the IRF-1 gene - considered to be the putative resistant gene in this cohort of ESN - revealed an association between the $619 \mathrm{~A}$ and $6516 G$ SNPs and protection against HIV infection. The presence of these SNPs was shown to result in a reduced level of the IRF-1 protein expression. IRF-1 may act as a Tat-independent initiator of the transcription of HIV genes following its binding to an ISRElike sequence of the HIV 5'-long terminal repeat, these results therefore suggest that a reduced IRF-1 expression down-regulates HIV transcription and replication at the initial stages of infection allowing the elicitation of an effective immune response [84].

Cytokines are crucial players in modulating the immune system and in counteracting pathogens. However, the results of analyses of polymorphisms in cytokines or cytokine receptors genes are often controversial. Interestingly, the majority of these analyses shows associations not with resistance, but rather with enhanced susceptibility to HIV disease. The most important of such associations are the following: (i) IL-2: the +3896 SNP results in accelerated disease progression [85]; (ii) IL-4 and IL-10: the 590T (IL-4) and the $-1082 G$ (IL-10) alleles, associated with higher protein levels, are more frequent in HIV infected compared to healthy individuals [86, 87]; (iii) IL-18: the $-607 C$ polymorphism correlates with increased risk of infection in a Brazilian paediatric cohort [88]; (iv) IFNAR1: the IFNAR1_18339 and IFNAR1_30127 gene polymorphisms correlate with susceptibility and disease progression to HIV infection [89]. Finally, the ILRa-2134 SNP, found with high frequency in a French cohort of slow progressors [90], is worthwhile mentioning as an example of one of the rare cytokine polymorphisms that seems to be associated with a positive effect on HIV disease. (Table 4).

Another intriguing HIV genetic resistant factor has been recently proposed [91]. The background for this work stems from analyses of the genetic correlates of antiviral immune responses against retroviral infections in animal models. In particular, it is known that the Rfv-3 locus on mouse chromosome 15 plays a pivotal role in modulating immune-mediated susceptibility to infection and disease outcome in the Friend Virus (FV) infection model [92-94]. The possible contribution of a putative human hortolog of the Rfv-3 locus to resistance against HIV infection was investigated in ESN by genotyping analyses of microsatellite markers on polymorphic loci spanning a region of human chromosome 22 (22q12-13) that is syntenic to mouse chromosome 15. Results showed that the allele 229 of the D22S423 microsatellite and the $156 / 158$ alleles at the D22S277 locus are significantly more expressed in ESN. Moreover, a disruption in linkage disequilibrium interesting the D22S276 locus was observed in the ESN alone. This last result suggests that a recombination event or a mutation occurred in the region surrounding the D22S276 locus in ESN individuals alone [91]. These data support the initial hypothesis that this region could be accounted for the HIV-specific immune response of ESN. Further investigations on this genetic correlate of resistance are in progress at present. Preliminary and very promising results allowed the identification of a 
particular haplotype, located within the regulatory region of an immuno-modulatory gene that is more frequent in ESN than in HIV-infected individuals. This finding supports the hypothesis that the gene harbouring this haplotype is the putative resistant gene in this cohort of ESN. The mechanism(s) by which this resistant gene exerts its protective effect is currently under investigation; the first observations seem to implicate a role for chemotaxis and immunological synapses formation processes. Moreover, the same genetic factor was found to be associated with resistance in Thai and African cohorts of ESN suggesting that this factor could be accounted for a more general mechanism of defence against HIV infection [manuscript in preparation].

\section{Conclusions}

Attempts to define the ' $\mathrm{X}$ ' factor associated with resistance to infection in HIV exposed seronegative individuals - and with delayed progression to AIDS in HIV-infected patients - have produced a huge amount of data that have been briefly summarized in this review. None of the factor analysed so far, though, can be unequivocally accounted for the observed resistance to HIV disease.

Critical features of this kind of research, such as inaccurate selection or stratification of patients and inadequate statistical analyses that do not consider, for example, correction for multiple tests as well as the often problematic reproducibility of the data in independent cohorts, can partially explain failure to identify such ' $\mathrm{X}$ ' factor. Moreover, most of the results presented herein are based on the candidate-gene approach, which imply an a priori knowledge of the gene function in HIV pathogenesis [95]. Nowadays, an alternative approach is represented by the genomewide association that, taking advantage of the information reported in the international haplotype mapping project (HapMap), allows to set up gene chips for the simultaneous study of hundreds of thousands polymorphisms. The first data based on this approach have in part confirmed the results described above, but have also highlighted new promising variants in genes never considered so far [96, 97].
The simplest way to justify the fact that we still do not know how HIV infection is modulated is, nevertheless, that HIV infection is an extremely complex disease, highlighting, once again that any single factor will unlikely be responsible for a phenomenon as complex as resistance to HIV-infection.

\section{Conflict of interest statement}

The authors do not have any conflicts of interest to declare.

\section{Acknowledgements}

We are grateful to Dr Valentina Naddeo for critically reviewing this article. Supported by grants from the Istituto Superiore di Sanita' 'Programma Nazionale di Ricerca sull' AIDS'; the EMPRO and AVIP EC WP6 Projects; the nGIN and GISHEAL EC WP7 Projects; the Japan Health Science Foundation; 2007 Ricerca Finalizzata [Italian Ministry of Health]; 2007 Ricerca Corrente [Italian Ministry of Health] and progetto FIRB RETI, Rete Italiana Chimica Farmaceutica CHEM-PROFARMA-NET [RBPR05NWWC].

\section{References}

1 Lo Caputo S, Trabattoni D, Vichi F et al. Mucosal and systemic HIV-1-specific immunity in HIV-1-exposed but uninfected heterosexual men. AIDS 2003; 17: 531-9.

2 Castelletti E, Lo Caputo S, Kuhn L et al. The mucosae-associated epithelial chemokine (MEC/CCL28) modulates immunity in HIV infection. PLoS ONE 2007; 2: e969.

3 Walker BD. Elite control of HIV Infection: implications for vaccines and treatment. Top HIV Med 2007; 15: 134-6.

4 Birch MR, Learmont JC, Dyer WB et al. An examination of signs of disease progression in survivors of the Sydney Blood Bank Cohort (SBBC). J Clin Virol 2001; 22: 263-70.

5 Goudsmit J, Bogaards JA, Jurriaans S et al. Naturally HIV-1 seroconverters with lowest viral load have best prognosis, but in time lose control of viraemia. AIDS 2002; 16: 791-3.

6 Piacentini L, Fenizia C, Naddeo V et al. Not just sheer luck! Immune correlates of protection against HIV-1 infection. Vaccine 2008; 26: 3002-7.

7 Samson M, Libert F, Doranz BJ et al. Resistance to HIV-1 infection in caucasian individuals bearing mutant alleles of the CCR-5 chemokine receptor gene. Nature 1996; 382: 722-5.

8 Liu R, Paxton WA, Choe S et al. Homozygous defect in HIV-1 coreceptor accounts for resistance of some multiply-exposed individuals to HIV-1 infection. Cell 1996; 86: 367-77. 
9 O'Brien SJ, Nelson GW. Human genes that limit AIDS. Nat Genet 2004; 36: 565-74.

10 Oppermann M. Chemokine receptor CCR5: insights into structure, function, and regulation. Cell Signal 2004; 16: 120110.

11 Rana S, Besson G, Cook DG et al. Role of CCR5 in infection of primary macrophages and lymphocytes by macrophage-tropic strains of human immunodeficiency virus: resistance to patientderived and prototype isolates resulting from the delta ccr5 mutation. J Virol 1997; 71: 3219-27.

12 Dean M, Carrington M, Winkler C et al. Genetic restriction of HIV-1 infection and progression to AIDS by a deletion allele of the CKR5 structural gene. Hemophilia Growth and Development Study, Multicenter AIDS Cohort Study, Multicenter Hemophilia Cohort Study, San Francisco City Cohort, ALIVE Study. Science 1996; 273: 1856-62.

13 Meyer L, Magierowska M, Hubert JB et al. Early protective effect of CCR-5 delta 32 heterozygosity on HIV-1 disease progression: relationship with viral load. The SEROCO Study Group. AIDS 1997; 11: F73-8.

$14 \mathrm{Wu}$ L, LaRosa $\mathrm{G}$, Kassam $\mathrm{N}$ et al. Interaction of chemokine receptor CCR5 with its ligands: multiple domains for HIV-1 gp120 binding and a single domain for chemokine binding. J Exp Med 1997; 186: 1373-81.

15 Smith MW, Dean M, Carrington M et al. CCR5-delta 32 gene deletion in HIV-1 infected patients. Lancet 1997; 350: $741-2$.

16 Stewart GJ, Ashton LJ, Biti RA et al. Increased frequency of CCR-5 delta 32 heterozygotes among long-term non-progressors with HIV-1 infection. The Australian Long-Term Non-Progressor Study Group. AIDS 1997; 11: 1833-8.

17 Cohen OJ, Paolucci S, Bende SM et al. CXCR4 and CCR5 genetic polymorphisms in long-term nonprogressive human immunodeficiency virus infection: lack of association with mutations other than CCR5-Delta32. J Virol 1998; 72: 6215-7.

18 Balotta C, Bagnarelli P, Violin M et al. Homozygous delta 32 deletion of the CCR-5 chemokine receptor gene in an HIV1-infected patient. AIDS 1997; 11: F67-71.

19 Theodorou I, Meyer L, Magierowska M et al. HIV-1 infection in an individual homozygous for CCR5 delta 32. Seroco Study Group. Lancet 1997; 349: 1219-20.

20 O'Brien TR, Winkler C, Dean M et al. HIV-1 infection in a man homozygous for CCR5 delta 32. Lancet 1997; 349: 1219.

21 Biti R, Ffrench R, Young J et al. HIV-1 infection in an individual homozygous for the CCR5 deletion allele. Nat Med 1997; 3: 252-3.

22 Heiken H, Becker S, Bastisch I et al. HIV-1 infection in a heterosexual man homozygous for CCR-5 delta32. AIDS 1999; 13: 529-30.

23 Sheppard HW, Celum C, Michael NL et al. HIV-1 infection in individuals with the CCR5-Delta32/Delta32 genotype: acquisition of syncytium-inducing virus at seroconversion. $J$ Acquir Immune Defic Syndr 2002; 29: 307-13.

24 Kuipers H, Workman C, Dyer W et al. An HIV-1-infected individual homozygous for the CCR-5 delta32 allele and the SDF-1 3'A allele. AIDS 1999; 13: 433-4.
25 Martinson JJ, Chapman NH, Rees DC et al. Global distribution of the CCR5 gene 32-basepair deletion. Nat Genet 1997; 16: 100-3.

26 Pereyra F, Addo MM, Kaufmann DE et al. Genetic and immunologic heterogeneity among persons who control HIV infection in the absence of therapy. J Infect Dis 2008; 197: 563-71.

27 Arenzana-Seisdedos F, Parmentier M. Genetics of resistance to HIV infection: role of co-receptors and co-receptor ligands. Semin Immunol 2006; 18: 387-403.

28 Gonzalez E, Bamshad M, Sato N et al. Race-specific HIV-1 disease-modifying effects associated with CCR5 haplotypes. Proc Natl Acad Sci U S A 1999; 96: 12004-9.

29 Mummidi S, Bamshad M, Ahuja SS et al. Evolution of human and non-human primate $\mathrm{CC}$ chemokine receptor 5 gene and mRNA. Potential roles for haplotype and mRNA diversity, differential haplotype-specific transcriptional activity, and altered transcription factor binding to polymorphic nucleotides in the pathogenesis of HIV-1 and simian immunodeficiency virus. J Biol Chem 2000; 275: 18946-61.

30 Jang DH, Choi BS, Kim SS. The effects of RANTES/CCR5 promoter polymorphisms on HIV disease progression in HIVinfected Koreans. Int J Immunogenet 2008; 35: 101-5.

31 Martin MP, Dean M, Smith MW et al. Genetic acceleration of AIDS progression by a promoter variant of CCR5. Science 1998; 282: 1907-11.

32 McDermott DH, Zimmerman PA, Guignard F et al. CCR5 promoter polymorphism and HIV-1 disease progression. Multicenter AIDS Cohort Study (MACS). Lancet 1998; 352: 866-70.

33 Salkowitz JR, Bruse SE, Meyerson H et al. CCR5 promoter polymorphism determines macrophage CCR5 density and magnitude of HIV-1 propagation in vitro. Clin Immunol 2003; 108: 234-40.

34 Hladik F, Liu H, Speelmon E et al. Combined effect of CCR5-Delta32 heterozygosity and the CCR5 promoter polymorphism $-2459 \mathrm{~A} / \mathrm{G}$ on CCR5 expression and resistance to human immunodeficiency virus type 1 transmission. J Virol 2005; 79: 11677-84.

35 Smith MW, Carrington M, Winkler C et al. CCR2 chemokine receptor and AIDS progression. Nat Med 1997; 3: 1052-3.

36 Anzala AO, Ball TB, Rostron T et al. CCR2-64I allele and genotype association with delayed AIDS progression in African women. University of Nairobi Collaboration for HIV Research. Lancet 1998; 351: 1632-3.

37 Faure S, Meyer L, Costagliola D et al. Rapid progression to AIDS in HIV+ individuals with a structural variant of the chemokine receptor CX3CR1. Science 2000; 287: 2274-7.

38 Vidal F, Viladés C, Domingo P et al. Chemokines LTNP Study Group. Spanish HIV-1-infected long-term nonprogressors of more than 15 years have an increased frequency of the CX3CR1 249I variant allele. J Acquir Immune Defic Syndr 2005; 40: 527-31.

39 Vasilescu A, Terashima Y, Enomoto M et al. A haplotype of the human CXCR1 gene protective against rapid disease progression in HIV-1+ patients. Proc Natl Acad Sci U S A 2007; 104: 3354-9.

40 Meddows-Taylor S, Martin DJ, Tiemessen CT. Reduced expression of interleukin-8 receptors $\mathrm{A}$ and $\mathrm{B}$ on polymorphonuclear 
neutrophils from persons with human immunodeficiency virus type 1 disease and pulmonary tuberculosis. J Infect Dis 1998; 177: 921-30.

41 Cocchi F, DeVico AL, Garzino-Demo A et al. Identification of RANTES, MIP-1 alpha, and MIP-1 beta as the major HIV-suppressive factors produced by CD8+ T cells. Science 1995; 270: 1811-5.

42 Zagury D, Lachgar A, Chams V et al. C-C chemokines, pivotal in protection against HIV type 1 infection. Proc Natl Acad Sci U S A 1998; 95: 3857-61.

43 Gallo RC, Garzino-Demo A, DeVico AL. HIV infection and pathogenesis: what about chemokines? J Clin Immunol 1999; 19: 293-9.

44 Paxton WA, Liu R, Kang S et al. Reduced HIV-1 infectability of CD4+ lymphocytes from exposed-uninfected individuals: association with low expression of CCR5 and high production of beta-chemokines. Virology 1998; 244: 66-73.

45 Koning FA, Jansen CA, Dekker J et al. Correlates of resistance to HIV-1 infection in homosexual men with high-risk sexual behaviour. AIDS 2004; 18: 1117-26.

46 Liu H, Chao D, Nakayama EE et al. Polymorphism in RANTES chemokine promoter affects HIV-1 disease progression. Proc Natl Acad Sci U S A 1999; 96: 4581-5.

47 Wichukchinda N, Nakayama EE, Rojanawiwat A et al. Protective effects of IL4-589T and RANTES-28G on HIV-1 disease progression in infected Thai females. AIDS 2006; 20: 189-96.

48 McDermott DH, Beecroft MJ, Kleeberger CA et al. Chemokine RANTES promoter polymorphism affects risk of both HIV infection and disease progression in the Multicenter AIDS Cohort Study. AIDS 2000; 14: 2671-8.

49 An P, Nelson GW, Wang L et al. Modulating influence on HIV/AIDS by interacting RANTES gene variants. Proc Natl Acad Sci U S A 2002; 99: 10002-7.

50 Gonzalez E, Kulkarni $\mathrm{H}$, Bolivar $\mathrm{H}$ et al. The influence of CCL3L1 gene-containing segmental duplications on HIV1/AIDS susceptibility. Science 2005; 307: 1434-40.

51 Modi WS, Lautenberger J, An P et al. Genetic variation in the CCL18-CCL3-CCL4 chemokine gene cluster influences HIV Type 1 transmission and AIDS disease progression. Am J Hum Genet 2006; 79: 120-8.

52 Modi WS, Goedert JJ, Strathdee S et al. MCP-1-MCP-3-Eotaxin gene cluster influences HIV-1 transmission. AIDS 2003; 17: 2357-65.

53 Winkler C, Modi W, Smith MW et al. Genetic restriction of AIDS pathogenesis by an SDF-1 chemokine gene variant. ALIVE Study, Hemophilia Growth and Development Study (HGDS), Multicenter AIDS Cohort Study (MACS), Multicenter Hemophilia Cohort Study (MHCS), San Francisco City Cohort (SFCC). Science 1998; 279: 389-93.

54 Reiche EM, Watanabe MA, Bonametti AM et al. Stromal cellderived factor 1 (SDF1) genetic polymorphism in a sample of healthy individuals, seronegative individuals exposed to human immunodeficiency virus type 1 (HIV-1) and patients infected with HIV-1 from the Brazilian population. Int $J$ Immunogenet 2006; 33: 127-33.
55 Ioannidis JP, Rosenberg PS, Goedert JJ et al. International meta-analysis of HIV host genetics. Effects of CCR5-Delta32, CCR2-64I, and SDF-1 3'A alleles on HIV-1 disease progression: an international meta-analysis of individual-patient data. Ann Intern Med 2001; 135: 782-95.

56 Carrington M, O'Brien SJ. The influence of HLA genotype on AIDS. Апnи Rev Med 2003; 54: 535-51.

57 Carrington M, Nelson GW, Martin MP et al. HLA and HIV-1: heterozygote advantage and $\mathrm{B} * 35-\mathrm{CW}^{*} 04$ disadvantage. Science 1999; 283: 1748-52.

58 Tang J, Costello C, Keet IP et al. HLA class I homozygosity accelerates disease progression in human immunodeficiency virus type 1 infection. AIDS Res Hum Retroviruses 1999; 15: 317-24.

59 Klein MR, van der Burg SH, Hovenkamp E et al. Characterization of HLA-B57-restricted human immunodeficiency virus type 1 Gag- and RT-specific cytotoxic T lymphocyte responses. J Gen Virol 1998; 79: 2191-201.

60 Goulder PJ, Bunce M, Krausa P et al. Novel, cross-restricted, conserved, and immunodominant cytotoxic T lymphocyte epitopes in slow progressors in HIV type 1 infection. AIDS Res Hum Retroviruses 1996; 12: 1691-8.

61 Gillespie GM, Kaul R, Dong T et al. Cross-reactive cytotoxic T lymphocytes against a HIV-1 p24 epitope in slow progressors with $\mathrm{B} * 57$. AIDS 2002; 16: 961-72.

62 Goulder PJ, Phillips RE, Colbert RA et al. Late escape from an immunodominant cytotoxic T-lymphocyte response associated with progression to AIDS. Nat Med 1997; 3: 212-7.

63 Kelleher AD, Long C, Holmes EC et al. Clustered mutations in HIV-1 gag are consistently required for escape from HLA-B27restricted cytotoxic T lymphocyte responses. J Exp Med 2001; 193: 375-86.

64 Flores-Villanueva PO, Yunis EJ, Delgado JC et al. Control of HIV-1 viremia and protection from AIDS are associated with HLA-Bw4 homozygosity. Proc Natl Acad Sci U S A 2001; 98: $5140-5$.

65 Martin MP, Gao X, Lee JH et al. Epistatic interaction between KIR3DS1 and HLA-B delays the progression to AIDS. Nat Genet 2002; 31: 429-34.

66 Lambotte O, Boufassa F, Madec Y et al. SEROCO-HEMOCO Study Group. HIV controllers: a homogeneous group of HIV1 -infected patients with spontaneous control of viral replication. Clin Infect Dis 2005; 41: 1053-6.

67 Migueles SA, Sabbaghian MS, Shupert WL et al. HLA B*5701 is highly associated with restriction of virus replication in a subgroup of HIV-infected long term nonprogressors. Proc Natl Acad Sci U S A 2000; 97: 2709-14.

68 Lockett SF, Robertson JR, Brettle RP et al. Mismatched human leukocyte antigen alleles protect against heterosexual HIV transmission. J Acquir Immune Defic Syndr 2001; 27: 277-80.

69 Dorak MT, Tang J, Penman-Aguilar A et al. Transmission of HIV-1 and HLA-B allele-sharing within serodiscordant heterosexual Zambian couples. Lancet 2004; 363: 2137-9.

70 MacDonald KS, Embree J, Njenga S et al. Mother-child class I HLA concordance increases perinatal human immunodeficiency virus type 1 transmission. $J$ Infect Dis 1998; 177: 551-6. 
71 MacDonald KS, Fowke KR, Kimani J et al. Influence of HLA supertypes on susceptibility and resistance to human immunodeficiency virus type 1 infection. J Infect Dis 2000; 181: 1581-9.

72 MacDonald KS, Embree JE, Nagelkerke NJ et al. The HLA A2/6802 supertype is associated with reduced risk of perinatal human immunodeficiency virus type 1 transmission. $J$ Infect Dis 2001; 183: 503-6.

73 Chiu YL, Greene WC. The APOBEC3 cytidine deaminases: an innate defensive network opposing exogenous retroviruses and endogenous retroelements. Апnи Rev Immunol 2008; 26: 317 53.

74 An P, Bleiber G, Duggal P et al. APOBEC3G genetic variants and their influence on the progression to AIDS. J Virol 2004; 78: 11070-6.

75 Valcke HS, Bernard NF, Bruneau J et al. APOBEC3G genetic variants and their association with risk of HIV infection in highly exposed Caucasians. AIDS 2006; 20: 1984-6.

76 Mariani R, Chen D, Schröfelbauer B et al. Species-specific exclusion of APOBEC $3 \mathrm{G}$ from HIV-1 virions by Vif. Cell 2003; 114: 21-31.

77 Biasin M, Piacentini L, Lo Caputo S et al. Apolipoprotein B mRNA-editing enzyme, catalytic polypeptide-like 3G: a possible role in the resistance to HIV of HIV-exposed seronegative individuals. J Infect Dis 2007; 195: 960-4.

78 Jin X, Brooks A, Chen $\mathrm{H}$ et al. APOBEC3G/CEM15 (hA3G) mRNA levels associate inversely with human immunodeficiency virus viremia. $J$ Virol 2005; 79: 11513-6.

79 Nisole S, Stoye JP, Saïb A. TRIM family proteins: retroviral restriction and antiviral defence. Nat Rev Microbiol 2005; 3: 799-808.

80 Speelmon EC, Livingston-Rosanoff D, Li SS et al. Genetic association of the antiviral restriction factor TRIM5alpha with human immunodeficiency virus type 1 infection. $J$ Virol 2006; 80: 2463-71.

81 Javanbakht H, An P, Gold B et al. Effects of human TRIM5alpha polymorphisms on antiretroviral function and susceptibility to human immunodeficiency virus infection. Virology 2006; 354: $15-27$

82 van Manen D, Rits MA, Beugeling C et al. The effect of Trim5 polymorphisms on the clinical course of HIV-1 infection. PLoS Pathog 2008; 4: e18.

83 Goldschmidt V, Bleiber G, May M et al. The Swiss HIV Cohort Study. Role of common human TRIM5alpha variants in HIV-1 disease progression. Retrovirology 2006; 3: 54.

84 Ball TB, Ji H, Kimani J et al. Polymorphisms in IRF-1 associated with resistance to HIV-1 infection in highly exposed uninfected Kenyan sex workers. AIDS 2007; 21: 1091-101.

85 Shrestha S, Strathdee SA, Galai N et al. Behavioral risk exposure and host genetics of susceptibility to HIV-1 infection. J Infect Dis 2006; 193: 16-26.
86 Konenkov VI, Smol'nikova MV. Polymorphism of promotor sites of interleukins- 4 and -10 and tumor necrosis factor-alpha genes in HIV-infected patients. Bull Exp Biol Med 2002; 133: 389-91.

87 Biasin M, Boasso A, Piacentini L et al. IL-4 and CXCR4 upregulation in HIV-infected and uninfected individuals from Maharashtra-Mumbai. AIDS 2003; 17: 1563-5.

88 Segat L, Bevilacqua D, Boniotto $\mathrm{M}$ et al. IL-18 gene promoter polymorphism is involved in HIV-1 infection in a Brazilian pediatric population. Immunogenetics 2006; 58: 471-3.

89 Diop G, Hirtzig T, Do $\mathrm{H}$ et al. Exhaustive genotyping of the interferon alpha receptor 1 (IFNAR1) gene and association of an IFNAR1 protein variant with AIDS progression or susceptibility to HIV-1 infection in a French AIDS cohort. Biomed Pharmacother 2006; 60: 569-77.

90 Do H, Vasilescu A, Diop G et al. Associations of the IL2Ralpha, IL4Ralpha, IL10Ralpha, and IFN (gamma) R1 cytokine receptor genes with AIDS progression in a French AIDS cohort. Immunogenetics 2006; 58: 89-98.

91 Kanari Y, Clerici M, Abe H et al. Genotypes at chromosome 22q12-13 are associated with HIV-1-exposed but uninfected status in Italians. AIDS 2005; 19: 1015-24.

92 Doig D, Chesebro B. Anti-Friend virus antibody is associated with recovery from viremia and loss of viral leukemia cellsurface antigens in leukemic mice. Identification of Rfv-3 as a gene locus influencing antibody production. J Exp Med 1979; 150: $10-9$.

93 Hasenkrug KJ, Valenzuela A, Letts V et al. Chromosome mapping of Rfv3, a host resistance gene to Friend murine retrovirus. J Virol 1995; 69: 2617-20.

94 Super HJ, Hasenkrug KJ, Simmons S et al. Fine mapping of the Friend retrovirus resistancegene, Rfv3, on mouse chromosome 15. J Virol 1999; 73: 7848-52.

95 Telenti A, Goldstein DB. Genomics meets HIV-1. Nat Rev Microbiol 2006; 4: 865-73.

96 Fellay J, Shianna KV, Ge D et al. A whole-genome association study of major determinants for host control of HIV-1. Science 2007; 317: 944-7.

97 Loeuillet C, Deutsch S, Ciuffi A et al. In vitro whole-genome analysis identifies a susceptibility locus for HIV-1. PLOS Biol 2008; 6: e32.

98 Miyazawa M, Lopalco L, Mazzotta F et al. The "immunologic advantage" of HIV-exposed sieronegative individuals. AIDS 2008 (in press).

Correspondence: Professor Mario Clerici, MD, Department of Biomedical Sciences and Technologies, University of Milan, Via Flli Cervi 93, 20090 Segrate (Milan), Italy.

(fax: +390250319677; e-mail: mario.clerici@unimi.it) 